\title{
ELECTRON CORRELATION IN ATOMS FROM PHOTOELECTHON SPECTROSCOPY
}

D. A. Shirley, S.-T. Lee, S. Süzer, R. L. Martin, E. Matthias, and R. A. Rosenberg

Niaterials Molecular Research Division, LBL and

Dept. of Chem., U. of California, Berkeley, CA 94720

\section{ABSTRACT}

The photoelectron spectrum of an atom $A$ ylelds the eigenenergies of the ion $\mathrm{A}^{+}$. Additional satellite peaks ariso because of electron correlation effects. Peaks observed in spectra from $\mathrm{Ca}, \mathrm{Sr}, \mathrm{Zn}, \mathrm{Cd}$, and $\mathrm{Hg}$ have been attributed to inftial-state configuration interaction, while final-state configuration interaction gives satellite peaks In a Ba specirum. The neon 1s "shakeup" spectrum is a result of both effects.

\section{INTRODUCTION}

The photoelectric effect has been known since approximately the turn of the century. EInstein 1 explained it in 1905 in the work that was to be noted in his Nobel Prize citation. Nevertheless, for over a half century thereafter this effect was known mainly as a threshold phenomenon manifested as the work function in metals or as the ionization potential in molecules.

Only in the 1960 's was photoelectron spectroscopj exploited as an experfmental method of practical use in many laburatorles. Its popularization followed the development of high-resolution electron spectrometers together with convenient line sources. 2,3 For Its first decade, photoelectron spectroscopy was divided into two rather distinct subdisciplines--ultraviolet photoelectron spectroscopy (UPS) and X-ray photoelectron spectroscopy (XPS or ESCA). With the adyent of intense variable-energy photon beans from electron storage rings, this artificial separation is now 
disappearing, and the generic term plotaelectron spectroscopy (PES) can be applied to the entire field.

The essential elements of a PES experiment are few and simp.le. A monochromatic photon beam of energy hv Impinges on a sample, ejecting electrons of kinetic energy

$$
T=h v-E_{B}
$$

Analysis of the electron intensity $N(T)$ yields peaks that can be assoclated with binding energies $E_{B}$ of varlous orbitals. In this way the complete one-electron orbital spectrum of a given element can be studied, and the Aufbanprinzip can be 1llustrated rather directly, a feature of particular appeal in atomir physics. Thus for example PES studies on the rare gas neon $\left(1 s^{2} 2 s^{2} p^{6} ; 1 s\right.$ ) will yleld the positions of the following states of $\mathrm{Ne}^{+}$:

$$
\begin{aligned}
& \left(1 s^{2} 2 s^{2} 2 p^{5} ;^{2} P_{3 / 2}\right) \\
& \left(1 s^{2} 2 s^{2} 2 p^{5} ;^{2} P_{1 / 2}\right) \\
& \left(1 s^{2} 2 s^{2} p^{6} ;^{2} s\right) \\
& \left(1 s 2 s^{2} 2 p^{6} ;^{2} s\right)
\end{aligned}
$$

awong others. Note that the ${ }^{2} S$ states are reached, as well as ${ }^{2} P$ states, from the $1_{S}$ ground states. We shall return to this point.

At this point one might well ask whether the PES method can yleld any information not already avallable from $X$-ray physics. After all, can one not simply build up these one-electron hole levels by comparing optical and $X$-ray data, as has been done in various tabulations of core electron binding energles?

The answer is (surprisingly) "No". The optical data vere obtained on free atoms, while $X$-ray spectro were taken using various condensed-phase anode materlals, usually metals. It is now well-known 4 that core-electron binding energies are systematically lower in metals than in free atoms. This follows from a combination of effects, the most important of which is usually "extra-atomic relaxation" or mobile valence electrons to shield the holes formed on photoemisston from core orbitals in metals. Table I illustrates the magnitude of this effect for the elements $\mathrm{Na}, \mathrm{N}$, and $\mathrm{Zn}$, taken from a new tabulation. 5 The difference,

$$
\mathbf{E}=\mathbf{E}_{\mathbf{B}}^{\mathbf{A}}-\mathbf{E}_{\mathbf{B}}^{\mathbf{M}}
$$


Table I. Binding energies of electronic

orbitals in acoms and metals.

\begin{tabular}{|c|c|c|c|c|}
\hline \multirow[b]{2}{*}{ Elenten } & N1 & $\mathbf{E}_{\mathbf{A}}^{A}$ & \multirow{2}{*}{$\begin{array}{l}\mathbf{E}_{\mathrm{B}}^{\mathrm{H}} \\
(\mathrm{eV})\end{array}$} & \multirow[t]{2}{*}{$\Delta \mathbf{B}$} \\
\hline & & & & \\
\hline \multirow[t]{3}{*}{$\mathrm{Na}$} & 18 & 1070 & 1074 & 5 \\
\hline & 28 & 71 & 66 & 5 \\
\hline & 38 & 5.1 & 3.5 & 1.6 \\
\hline \multirow[t]{2}{*}{ N1 } & $2 p_{3 / 2}$ & 869 & 859 & 10 \\
\hline & $3 p$ & 83 & 72 & 11 \\
\hline \multirow[t]{2}{*}{$\mathbf{2 n}$} & $2 p_{3 / 2}$ & 1029 & 1026 & 3 \\
\hline & $3 p$ & 96 & 93 & 3 \\
\hline
\end{tabular}

between atomic and wetallic core-level binding energles, runs from 3 to $11 \mathrm{eV}$ in thase elements, a abstantial effect Indeed.6 Thus we must conclude that all previous cabulations of core-level binding energies which did not distinguish between atomic and petallic samples are systemat ically in error.

Let us now compare and contrast PES with photon spectroscopy, referring to Fig. 1 . We consider an atom that has $s$ oymetry in a ground state, and ve work in the electric dipole approxiantion. In the presence of a radiation field represented by the vector potential $A(t)$, the electron momenta are replaced by $p-e / c$ standard time-dependent perturbacton theory wth conventional approximations yields a transition probabilicy governed by the matrix element

$$
M \text { e. a }\left(\Psi_{\mathrm{f}}|\mathrm{A} \cdot \mathrm{p}| \Psi_{1}\right)
$$

Only states of p-syumetry are excited. For low-energy photons this means that $P$ bound states of the N-electron (atontc) system can be found (shary 11nes. Fig. 1). Above the photolonization threshold, P-gyumetry K-electron atates are olso formed, thus aatisfying the electric dipole selection rule. These give absorption edges rather than sharp 1ines. Further, each of these $P$ states consists of a state of the ( $N-1$ ) electron (Ionic) aystem, of arbitrary orbital angular momenta L", plus a continuum electron in a stace of orbital angular momentum \& that eatisfies the triangle condition 


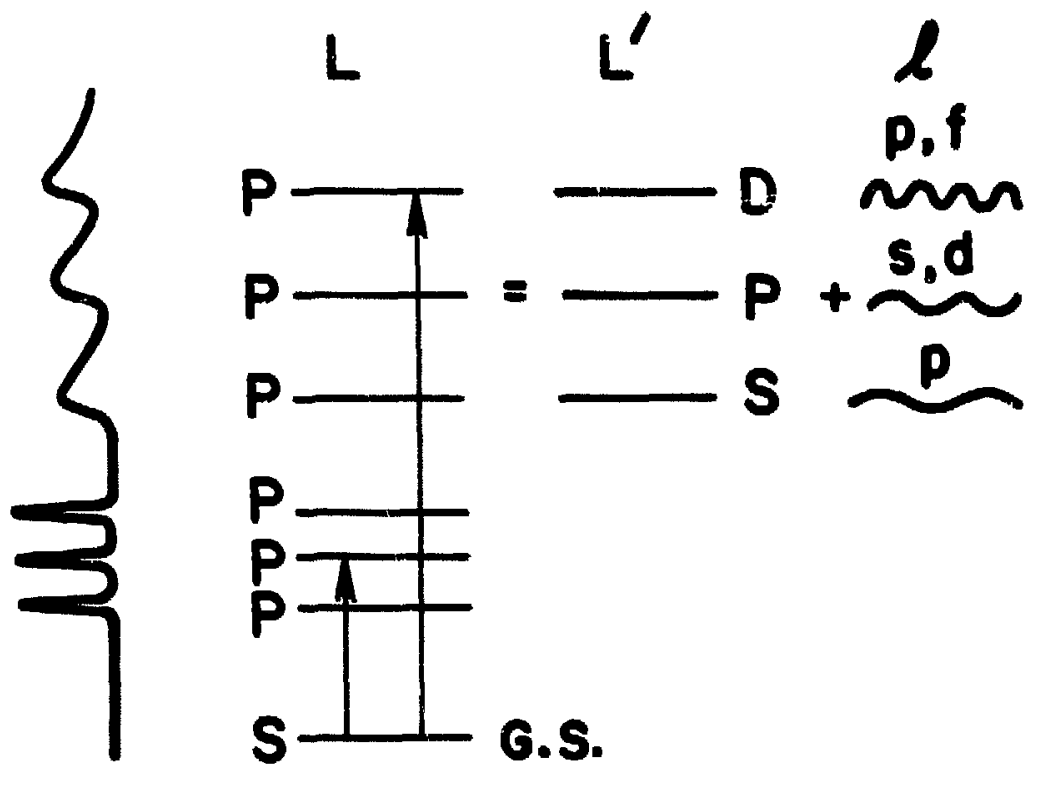

X9L768-3809

FIG. 1. LLUSTAMTICN OF TIE WAY IN MIICI THE ELECTRIC DIPOLE SELECTIOA RULES APPI,LED TO AN ATCA IN AN S STATE VIELDS

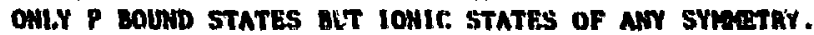




$$
\underline{\underline{L}}+\underline{q}=\underline{\underline{L}}
$$

with $|4|-1$ lor the $P$ state. Thus in ef fect there is no absolute oclecelon rule for photocmisston and all states are populated.

We now turn (rom onc-cleceron propertles (or apporent one electron propertieg) to a phenommon that 18 manifestly multielectronic in inture, nanely electron-electron correlation. For simplicity inf: fal-state correlation effects are trented in the next eection using the configuration-interaction formalism. Final-state corrclations are the subject of Section III. In Section IV the neon is satellttes, in which both Initial- and final-state correlations nre important, are discusaed briefly.

\section{INITIAL STATE CONFIGURATION INTERACTION}

A one-electron atow or Ion can be treaced exactly, but any mult 1- (even two-) electron system must be dealt vith using approxtmate methods. Thus the ground state of the can be described only roughly by the configuration $18^{2}$. The $e^{2} / r_{12}$ electronelectron repulsion cerm in the Hamilcostan may be regarded as leading to an explicit dependence of the electrontc vavefunction on $\tau_{12}$. It 16 computationally most conyenient to treat correlation through configuration interaction, 7 in which eigenatates are described by adding excited configurations with the correct amplitude and phase relations. Thus the ${ }^{\prime} \mathrm{s}$ ground state of He 18

$$
(1 s)=a\left(1 s^{2}\right)+b\left(2 s^{2}\right)+c\left(2 p^{2}\right)+\ldots
$$

In the alkaline-corth, or Group'IIA, atoms, the valence configuration way be represented as (for exwople)

$$
Y(C a)=a\left(4 s^{2}\right)+b\left(4 p^{2}\right)+c\left(3 d^{2}\right)+\ldots
$$

and alallarly for Sr and Ba.

Photocmission from these ground states woild yield only the

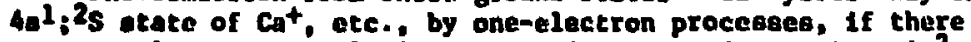
were no electron correlation. The adaixed configurations $4 p^{2}$. $3 d^{2}$. ete. Iend directly to observable $4 p^{1} q^{2} p$ and $3 d^{1} ;{ }^{2}$ final atater on photongisaton. with the line intensities beting related to adalxture coefficients in the initiul atates. Thus photoanieatoa spectro can give a rather direct messure of initial-state configuration internction (ISCI). Such apectra have now been observed in $\mathrm{Cn}, \mathrm{Sr}, \mathrm{Bn}, \mathrm{Zn}, \mathrm{Cd}$, and $\mathrm{Hg}_{8} 8-10$ The HeI resonance Inc at $21.2 \mathrm{eV}$ vas used to Induce photoemlasion in every case except Be, for which the NeI lines $(16.7,16.9 \mathrm{eV})$ vere ewployed. 
Table II lists the prominent satellites that were identified In the photoclectron spcecra of these atoms.

Comparisons with theory are st 111 in a rather crude stage. A quantitative analysis of these spectra requires both configuration Interaction calculations on the initial states and estimates of tho relative cross-sections for $4 \mathrm{~s}, 4 \mathrm{p}, 3 \mathrm{~d}$, etc., photoemisgion. The latter are not yet avallable. MulticonfiguratIon llartree-lock calculations were done by Kim and Bagusl]. and by Hansen. 12 The admixture coefficients that were obtained would predict the satellite intensities to be too weak by factors between 2 and 20 before cross-section effects are taken into account. It will be interesting to learn whether the cross-section variation will resolve this discrepancy.

Because the configuration-Interaction formalisw is just one approach to the electron correlation problem, the reality of the configuration admixtures can be questioned. After all, one need ncc in principle even use the CI picrure. The satellites would etill be observed: what would they then mean? The answer is that operationally the one-electron states in Ca+ (for example), together with the continuum final states are projected onto the initial state of Ca, through the dipole transition operator. This Is a general statement of the process. A more specific statement, In terms of ISCI, requires care in selecting the basis functions. Once a particular set of functions is chosen, however, there exists a unique answer as to how much a given function is admixed into the Initial state.

We conclude this section by pointing out a special case of ISCI involving spin-orbit coupling. The ground state of atomic $\mathrm{Pb}$ is normally $6 \mathrm{~s}^{2} 6 \mathrm{p}^{2}$., In strong spin-orbit coupling this becomes 13 mainly $6 s^{2} 6 p_{1 / 2}^{2}$ with a small admixture of $6 s^{2} 6 p_{3}^{2} / 2$. Sitzer 14 has shown that the photoemiosion spectrum of atomic $\mathrm{Pb}$ indeed shows a very intense $\left(6 s^{2} \mathrm{~b}_{1 / 2} ;{ }^{2} \mathrm{P}_{1 / 2}\right)$ peak and a much less (7X) intense $\left(6 s^{2} 6 \mathrm{p}_{3 / 2} i^{2} \mathrm{P}_{3 / 2}\right)$ peak, as expected. Also present were all the peaks expected to arise from admixtures of $\operatorname{sp}^{2} \mathrm{~d}$ and $B^{2} d^{2}$ Into the ground state.

\section{FINAL-STATE CONFIGURATION INTERACTION (FSCI)}

This phenowenon 18 manifested as additional setellites or very unusual intensities in a photoemission spectrun. There are scveral known cases of FSCI. Dne of the most dramatic is the HeI. spectrum of atomic bartum, which vas reported by Brehm and Höfleris and by Hotop and Mahr.16 Thls spectrum was re-discovered in our laboratory in the course of ISCI studies In alkaline earths. The 
Table II. Sumary of satellites attributable to ISCI in photoelectron spectra of Group II elements.

\begin{tabular}{|c|c|c|c|}
\hline Ion & Main Line & Satellites & References \\
\hline $\mathrm{Ca}\left(4 \mathrm{~s}^{2}\right)$ & 48 & $4 p, 3 d, 5 s, 4 d$ & 10 \\
\hline $\operatorname{sr}\left(5 s^{2}\right)$ & $5 s$ & $5 p *, 4 d, 6 s, 5 d, 4 f$ & 10 \\
\hline $\operatorname{Ba}\left(6 s^{2}\right)$ & 68 & $6 p *, 5 d *, 7 s, 6 d, 4 f$ & 10 \\
\hline $\operatorname{Zn}\left(3 d^{10} 4 s^{2}\right)$ & 48 & $4 p *$ & 10 \\
\hline $\operatorname{cd}\left(4 d^{10} 5 s^{2}\right)$ & 58 & $5 p *$ & 8,10 \\
\hline$H_{8}\left(5 d^{10} 6 s^{2}\right)$ & 68 & $6 p *$ & 9,10 \\
\hline
\end{tabular}

essential unusual features of the RaI (HeI) spectrun are the Breatly enhanced $5 d$ and $6 p$ peaks and a number of addictonal $\mathrm{nl}$ peaks of $\mathrm{Bat}^{\mathrm{t}}$ arising from resonant auto-ionizacion by the $21.2 \mathrm{eV}$ radiation. For example, the $6 f$ and $8 p$ states are clearly present.

The explanation for this phenomenon is too involved to be presented In detail here, but basically the HeI radiation resonantly excites a state of Ba that is embedded in the continuun of $\mathrm{Ba}^{+}$. The final N-electron state (N $=56$ in this case) can be regarded as consisting of interacting N-electron configurations, each of which is made up of an N- 1 electron atate of Bat coupled with an unbound electron in a continuum state. When the resonance condition is satisfied, the outgoing electrons carry kinetic energies $\left\{h v-E_{1}\right\}$, where $\left\{E_{1}\right\}$ are the energles of the Bat states.

In $\mathrm{Ba}$, autoionization occurs because of excitation of an electron from the $5 p^{6}$ shell to an ns or nd atate. Thus the total configuration of the state embedded in the continuum is $5 p^{5} 6 a^{2}$ (ns or $n d$ ). This way be loosely regarded as a Rydberg-11ke state below the 5p photolonization threshold In Ba. Proceeding upward In $Z$ from Ba through the rare earths, the 5p Ionization threshold does not Increase dramatically, because the additional nuclear charge is in large maasures shielded by the filling $4 f$ shell, and the Ion core potential changes only slowly. Thus resonant autolonization by the HeI $21.22 \mathrm{eV}$ line and associated satellite radiations persists through $S$ (FIg. 2) and $B u$ $(z=62,63)$. It has disappesed by $Y b(z=70)$. 


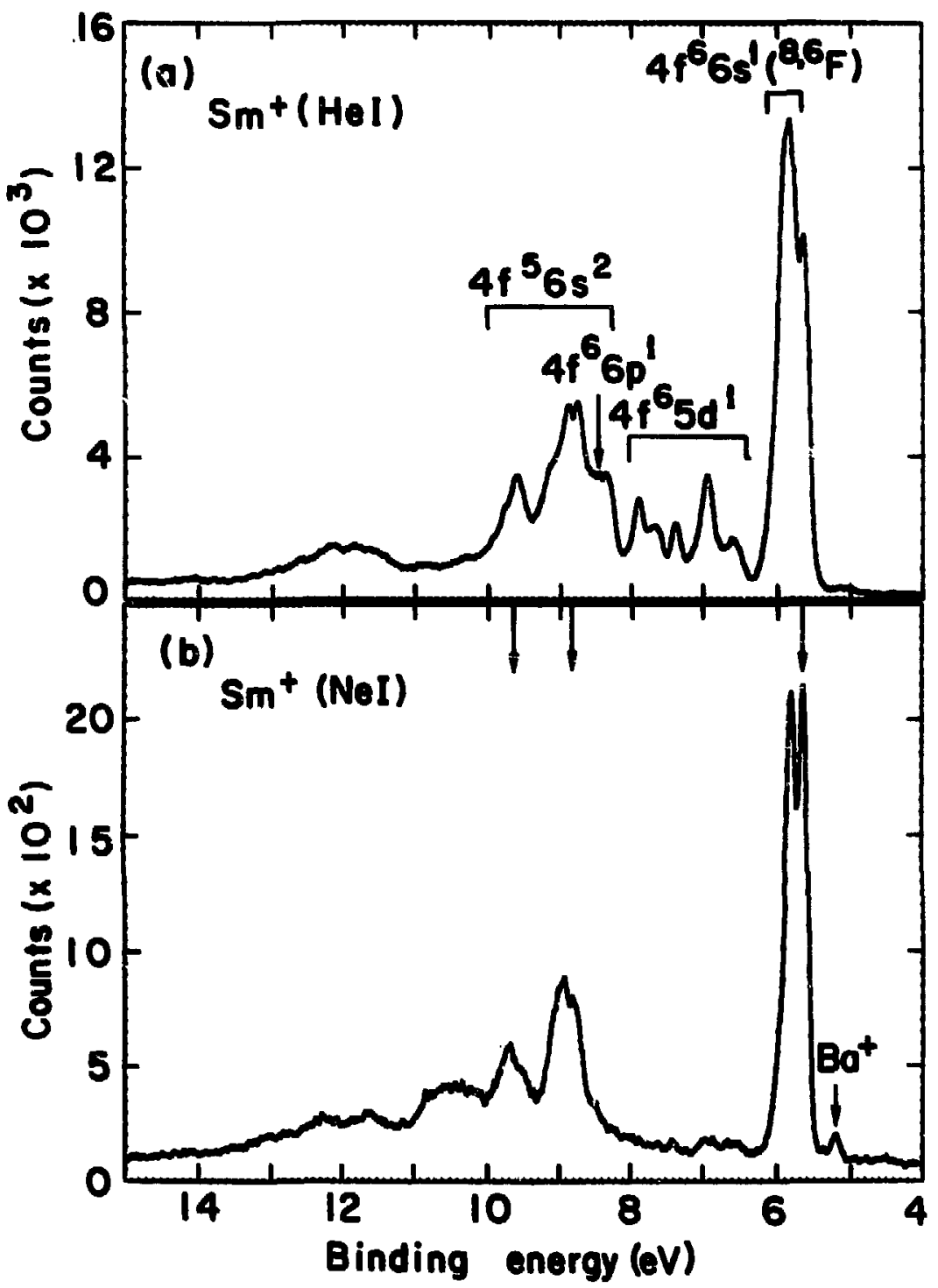

xal $7 \pi+20$

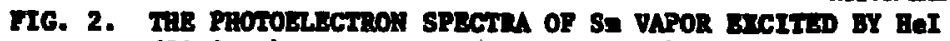
$(21.2 \circ V)$ AD BY NeI $(16.7,16.9 \mathrm{eV})$ BDIATION. THE

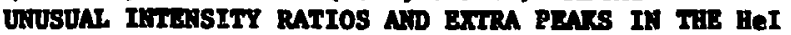
SFBCXRU ARISB FEOA AUTOLONIZATIOA FOLLOUING RBSOMNT BXCIYATIOA OF THE Sp SHBLL. 
Fano und Cooper 17 have discussed configuration interaction effects from a very general vjewpoint. As tunable (aynchrotron) radiation sources become available, many instances of these phenomena can be expected.

\section{THE Ne 1s SATELlites: ISCI AND FSCI}

As a Iinal example, let us consider the "shakeup" opectrua of the $\mathrm{Ne} 1 \mathrm{~s}$, In which both initial- and final-state correlations play a role. A high-resolution photoemission spectrum of the is region of Ne was reported by Gelius. 18 In addition to the many transition

$$
\mathrm{Nas}\left({ }^{1} s ; 1 s^{2} 2 s^{2} 2 p^{6}\right) \stackrel{-e}{\rightarrow} \mathrm{Ne}^{+}\left({ }^{2} s ; 1 s^{1} 2 s^{2} 2 p^{6}\right)
$$

at $870 \mathrm{eV}$, there was a large number of weak satellite 1 ines at about $40 \mathrm{eV}$ higher energy. These arise from transitions of the form

$$
\begin{aligned}
& \operatorname{Ne}\left({ }^{1} s ; 1 s^{2} 2 s^{2} 2 p^{6}\right) \rightarrow \mathrm{Ne}^{+}\left({ }^{2} s ; 1 s^{1} 2 s^{2} 2 p^{5} 3 p\right) \\
& \mathrm{Ne}^{+}\left({ }^{2} \mathrm{~s} ; 1 \mathrm{~s}^{1} 2 \mathrm{~s}^{2} 2 \mathrm{p}^{5} 4 \mathrm{p}\right) \\
& \mathrm{Ne}^{+}\left({ }^{2} \mathrm{~s} ; 1 \mathrm{~s}^{1} 2 \mathrm{p}^{2} 2 \mathrm{p}^{5} \mathrm{sp}\right) \\
& \cdot \\
& \text { - } \\
& \text { etc. }
\end{aligned}
$$

(It should be noted that eacli configuration on the right gives two final states because of exchange splitting.)

There are two ways in which these transition can be understood on the basis of FSCI alone. We note that a nonzero transition probability requires overlap between the "passive" electron configurations. Expanding the matrix element, for photoentesion,

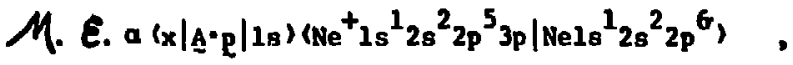

we see that the overlap product would vanish if the basis functions In $\mathrm{Ne}^{+}$and $\mathrm{Ne}$ were the same (because $(3 \mathrm{p} / 2 \mathrm{p}$ ) would be zero). In fact they are not: the $\mathrm{Ne}^{+} n=2$ functions are relaxed because of the added core charge, giving finite overlap. Alternatively we could imagine using the basis set in $\mathrm{Ne}^{+}$as in Ne, but describing the relaxation in terms of configuration mixing, again yielded finite overlap. This effect 1s, however, not readily separable 
from the second way in which FSCl leads to batellites; namely configuration interaction that is already present 1 rrespecilve of relaxation. After all, the "final" states In $\mathrm{Ne}^{+}$are in fact elgenstates of a liamiltonian with no reference to photoelectron spectroscopy.

The FSC:I effects in the Ne $1 \mathrm{~s}$ spectrum have been know for some time, but calculated spectra based on Fscl alone show much lower relative intensities in the satellites than are observed experimentally. It has been pointed out by Martin19 that ISCI is also imporzant in this case. In fact ISCI is responsible for about one-half the observed satellite intensities. There is a great deal of symmetry between the ISCI and FSCI contributions to the satellite intensities, because the $\mathrm{Ne}$ and $\mathrm{Ne}^{+}$states are basically quite similar, even at the level of electron correlations. Thus it even turns out that the satellice spectrua gives a fairly direct plcture of configuration admixing in the ground stace.

In conclusion, we have sought in this paper to summarize specific cases in which electron correlation has manifescly affected photoelectron spectra. It aeems likely that photoelectron spectroscopy will prove increasingly useful in elucidating problems of this nature in atomic physics.

\section{REFERENCES}

1. A. Einstein, Ann. d. Physik 17, 132 (1905).

2. K. Siegbahn, C. Nordling, A. Fahlman, R. Nordberg, K. Hamrin, J. Hedman, G. Johansson, T. Bergmark, S.-E. Karlsson, I. Lindgren, and B. J. Lindberg, ESCA-Atomic, Molecular, and Solid Stace Structure by Means of Electron Spectroscopy, Nova Acta Reglae Soc. Sc1. Upsallensis Ser. IV, vol. 20, 1967.

3. D. W. Turner, C. Baker, A. D. Baker, and C. R. Brundle, Volecular Photoelectron Spectroscopy (HLley Interscience, 1970).

4. L. Ley, S. P. Kowalczyk, F. R. McFeely, R. A. Pollak, and D. A. Shirluy, Phys. Rev, B E, 2392 (1973).

5. D. A. Shirley, Chem. Phys. Letters 16, 220 (1972).

6. This difference remains after the work function correction otherwise it would be even larger.

7. J. C. Slater, Quantum Theory of Atomic Stzucture (McGraw-Hill, 1960). vol. 11, P. 40 . 
8. S. Süzer and D. A. Sh1rley, J. Chem. Phys, 6], 2481 (1974).

9. J. Berkowitz, J. I., Dehmer, Y, K. K1m, and J. P. Desclaux, J. Chem. Phys. 61: 2556 (1974).

10. S. Süzer, S.-T. Lee, and D. A. Shtrley, Phys. Rev. A $\underline{13}_{0}$ 1842 (1976).

11. Y. K. Kim and P. S. Bagus, Phys. Rev. A ㅁ, 1739 (1973).

12. J\&rgen E. Hansen (private communication, June 1976).

13. E. U. Condon and G. $M$. Shortley, The Theory of Atomic Spectra (Cambridge University Press, 1935), F. 275.

14. S. Süzęr, M. S. Banna, and D. A. Shirley, J. Chem. Phys. 63, 3473 (1975).

15. B. Brehm and K. Höfler, Int. J. Mass Spectrom. Ion Phys. 17, 371 (1975).

16. H. Hotop and D. Mahr, J. Phys. B: Atom. Nolec. Phys. B, L301 (1975), and private communication.

17. U. Fano and J. W. Cooper, Rev. Mod. Phys. 40, 441 (1968).

18, U. Gellus, J. Rlectr. Spectr. and Rel. Phenomena 5, 985 (1974).

19. R. L. Martin and D. A. Shirley, Phys. Rev. A 13, 1475 (1976).

*Th1s work was done uith support from the U. S. Energy Research and Development Administration. 